\title{
2 Theoretischer Rahmen
}

Ausgehend von soziologischen und philosophischen Überlegungen zur Wissens- und Welterzeugung (Berger/Luckmann, Goodman) beschreibt dieses Kapitel den Beitrag von Erzählungen und Medien (Nünning/Nünning, Erll, Anderson) sowie von Stereotypen (Lippmann) zur Konstruktion von Wissen und Welten. Es wird ferner dargelegt, inwiefern die narrativen, medialen und stereotypen Modi der Wissens- und Welterzeugung für die Produktion, Speicherung und Zirkulation des kulturellen Wissens einer Gesellschaft gewichtige Komponenten darstellen und im Hinblick auf die Analyse der spectatorialen Geschlechterkonstruktionen eine geeignete Grundlage bilden.

\subsection{EINLEITENDE ÜBERLEGUNGEN ZUR WISSENS- UND WELTERzEUGUNG}

In den vergangenen Jahrhunderten gab es unzählige Versuche, die Welt zu erklären und das Wissen über die Welt in sinnhafter Weise zu beschreiben. In der Antike verabschiedet man sich vom mythischen Weltbild und orientiert sich am geozentrischen, das im 16. Jahrhundert mit den Beobachtungen von Kopernikus (1473-1543) allmählich vom heliozentrischen Weltbild abgelöst wird. Stets existier(t)en Weltbilder aber auch parallel zueinander, da auch religiöse und politische Weltauffassungen mit den astronomisch-wissenschaftlichen koexistieren: So beherrscht zunächst ein theozentrisches Weltbild das mittelalterliche Europa, das in der Renaissance allmählich in ein anthropozentrisches übergeht, in dem nun also nicht mehr Gott, sondern der (individuelle) Mensch in den Mittelpunkt rückt. Zwischen und auch innerhalb von Religionen existieren unterschiedliche Glaubensbekenntnisse gleichzeitig, wobei sich manche respektvoll, andere im erbitterten Kampf gegenüberstehen. Auch die Weltauffassungen der diversen politischen ,Ismen“ (Absolutismus, Parlamentarismus, Kommunismus etc.), die neben Ideologien auch Verhaltensvorschriften und Handlungspraktiken liefern, bestehen vielfach parallel zueinander; genauso wie die wissenschaftlichen Weltauffassungen aus den verschiedenen wissenschaftlichen Disziplinen wie der Philosophie oder der Mathematik. Gemein ist allen Weltbildern dabei, dass sie eine Vorstellung davon vermitteln, wie die Welt ,ist` und was in dieser Welt als ,normal' gilt, das heißt, Weltbilder spiegeln die Norm- und Normalitätsvorstellungen einer bestimmten Gruppe wider.

Aus dieser kurzen Schilderung wird bereits deutlich, dass weder, das ' Wissen über die Welt noch ,die' Welt an sich seit jeher schon als solche existieren, sondern dass 
Wissen und Welten auf spezifische Art und Weise ,realisiert` werden. Der sozialkonstruktivistischen Wissenssoziologie von Peter L. Berger und Thomas Luckmann (1991) zufolge entsteht Wissen über die Alltagswelt, das heißt über die alltägliche Wirklichkeit, durch Habitualisierung ${ }^{1}$ und Institutionalisierung. ${ }^{2}$ Aufgrund der Bezugnahmen auf Einzelpersonen und Gruppen kann das durch Habitualisierung generierte Wissen als individuelles und das durch Institutionalisierung generierte als kollektives Wissen charakterisiert werden (cf. Abels 2009, 75-79). In Anbetracht der Tatsache, dass individuelles wie kollektives Wissen von Kultur zu Kultur - ebenso wie von Epoche zu Epoche - variiert, kann auch von kulturellem Wissen gesprochen werden. Es erwächst aus habitualisierten und institutionalisierten Alltagspraktiken, Politik, Literatur, wissenschaftlichen Abhandlungen oder Bildern innerhalb eines Kulturkreises und bezeichnet „die Gesamtmenge der in einer Kultur zirkulierenden Kenntnisse, die durch Kommunikation, Erfahrung und Medien konstruiert, erworben und tradiert werden“ (Neumann/Nünning 2006, 13). Indem das kulturelle Wissen für eine Gruppe bestimmte gesellschaftlich prävalente Werte, Normen und Praktiken ${ }^{3}-$ das heißt bestimmte

1 Menschen machen im Laufe ihres Lebens die unterschiedlichsten Erfahrungen, und je häufiger sich diese ähneln, umso mehr werden sie als typisch wahrgenommen; umso fester brennen sie sich ins Gedächtnis ein. Aus typischen Erfahrungen bilden sich typische Erwartungen, die es ermöglichen, die Zukunft in gewisser Weise vorherzusagen - zumindest in Bezug auf sich wiederholende Erfahrungen. Menschen bauen also ein bestimmtes Vorwissen auf. Dabei wird jenes Verhalten, das wiederholt erfolgreich war, als ,typisches Verhalten' angesehen und verinnerlicht (habitualisiert) (cf. Abels 2009, 76-77).

2 Werden Gewohnheiten für eine größere Gruppe von Menschen bedeutsam, d. h. wird das Wissen um ,typische“ Verhaltensmuster also auf alle Mitglieder einer Gesellschaft übertragen, dann spricht man von der Institutionalisierung von Wissen. Der ständige Gebrauch dieser wechselseitig vorhersehbaren Austauschformen definiert gesellschaftliche Institutionen. Eine Institution ist ein oft wiederholtes Interaktionsmuster, bei dem die Beteiligten als Typen miteinander in Beziehung treten (cf. Abels 2009, 78-79).

3 ,Werte' sind allgemeine Vorstellungen darüber, was in einer Gesellschaft für wünschenswert erachtet wird. Sie dienen der Verhaltensorientierung und stellen gleichzeitig Maßstäbe zur Beurteilung von Verhalten bereit. $\mathrm{Zu}$ Werthaltungen zählen beispielsweise Meinungsfreiheit, Erhaltung der Gesundheit, Versorgung der Kranken, Ausbildung der Jugend, Gleichberechtigung, Glücksvorstellungen oder die Einstellung zum Umweltschutz. Damit Werte in einer Gesellschaft „,wirksam werden und dass sie in sozialen Situationen konkretisiert und sozusagen in Handlungsanweisungen übersetzt werden können, dafür sind soziale Normen hilfreich [kursiv im Orig.]“ (Vester 2009, 55; cf. Beckers 2016, 373-377).

,Soziale Normen' sind verhaltenssteuernde Regeln, die mehr oder weniger genau festlegen, was in einer bestimmten sozialen Situation eine angemessene und erwartete Verhaltensweise ist. Es handelt sich dabei um Erwartungen in Bezug auf das Handeln oder Nichthandeln von Gesellschaftsmitgliedern, auf das bei Missachtung negative Sanktionen folgen. Normen helfen Menschen, bei der Orientierung im Alltag, beim Abwägen des eigenen Handelns sowie bei der Erwartung und Einschätzung des Handelns von Anderen (cf. Tranow 2016, 256-260; Vester 2009, 55-56).

,Praktiken“ sind als routinisierte, körperliche Handlungen und Verhaltensweisen zu verstehen, die das Zusammenleben von Individuen und Gruppen innerhalb einer Gesellschaft 
Annahmen über die Welt und die Wirklichkeit - zur Verfügung stellt, hält es „einen reproduzierbaren Bestand möglicher Denk-, Orientierungs- und Handlungsmuster bereit, die innerhalb der jeweiligen kulturellen Rahmenbedingungen als gesellschaftlich gültig und wertvoll gelten“" (ibid.).

Der Philosoph Nelson Goodman geht in Ways of Worldmaking (Weisen der Welterzeugung) der Entstehung, Vielfalt und Wechselbeziehung von Weltversionen nach und betont, dass , ,[w]ir [...] bei allem, was beschrieben wird, auf Beschreibungsweisen beschränkt [sind]. Unser Universum besteht sozusagen aus diesen Weisen und nicht aus einer Welt oder aus Welten“ (Goodman 1984, 15). Zu diesen Beschreibungsweisen zählt er die folgenden fünf erweiter- und kombinierbaren Prozesse: (1) Komposition und Dekomposition, (2) Gewichtung, (3) Ordnen, (4) Tilgung und Ergänzung sowie (5) Deformation (cf. ibid., 20-30). Laut Goodman hängt das, ,[w]as gesagt wird, wie es gesagt wird, was ausgedrückt wird und wie - [...] eng zusammen und gehört zum Stil“ (ibid., 46). Ebenfalls räumt Goodman den Beobachtenden eine tragende Rolle in der Welterzeugung ein, da sie es sind, die in konstruktivistischer Manier verbal und non-verbal „,mittels Wörtern, Zahlen, Bildern, Klängen oder irgendwelchen anderen Symbolen ${ }^{4}$ in irgendeinem Medium solche Versionen erzeug[en]“" (ibid., 117). Goodman meint mit ,erzeugen' also nicht nur das Sprechen und Schreiben, sondern auch Malen, Musizieren, Tanzen etc. Neben den Wissenschaften fungieren für ihn demnach auch die Künste ganz klar ,als Modi der Entdeckung, Erschaffung und Erweiterung des Wissens“5 (ibid., 127) und sind an der Erzeugung von Weltversionen beteiligt.

strukturieren. Bereits Simone de Beauvoir betrachtet das Frausein als Produkt soziokultureller Praktiken, als sie davon spricht, dass man nicht als Frau geboren, sondern zu ihr gemacht werde. Der Begriff des ,Doing Gender` bringt den permanenten Herstellungsprozess von Geschlechtszugehörigkeit und -identität durch die Wiederholung von sozialen und kulturellen Praktiken auf den Punkt. Gleichzeitig verweist der Begriff des Doing Gender darauf, dass sich in der Beherrschung und Befolgung ,der entsprechenden Praktiken [...] die (geschlechtsbezogenene [sic]) Handlungskompetenz der Gesellschaftsmitglieder“ (Meuser 2010, 151) offenbart (cf. Gildemeister 2010).

4 Goodmans Symbolbegriff ist ein sehr allgemeiner, der - wie gerade angedeutet - Wörter, Texte, Töne, Diagramme oder Karten umfasst. Mit diesen Symbolen werden ,Versionen der Welt` erzeugt. Sie können unterschiedlichen Symbolsystemen wie den Wissenschaften, der Philosophie oder den Künsten zugeordnet werden, was bedeutet, dass es je nach Symbolsystem spezifische, Weisen der Welterzeugung ' und somit jeweils eigene Weltversionen gibt.

5 Wissen wird bei Goodman durch Verstehen ersetzt. Damit tritt anstelle der gängigen auf Aristoteles zurückgehenden Vorstellung von Wissen als endlos wachsender Anhäufung wahrer Aussagen, über die Gewissheit herrscht, ein aktiver Verstehensbegriff. Verstehen wird bei Goodman zu einem dynamischen Prozess, das im - ebenfalls dynamischen - Prozess der Welterzeugung in den jeweiligen Symbolsystemen generiert wird. Verstehen wird somit ,zu einem offenen Prozeß, der niemals abgeschlossen ist. Er ist ein aktiver, dynamischer Vorgang, er resultiert aus Probieren, Konstruieren und Testen. Ziele und Zwecke im Verstehensprozeß sind niemals endgültig festgelegt, sie können sich wandeln, abhängig von dem, was erreicht werden soll. Verstehen selbst kann sich so im Laufe der Zeit einer Entwicklung unterziehen und auf historische Veränderungen reagieren, [...]“ (cf. Ammon 2005, 318). 
Indem die Rolle und Fähigkeit, Weltversionen zu erzeugen, primär an den Menschen gebunden wird, kann der Mensch bereits als Medium der Welterzeugung konzipiert werden. Er ist es, der seit Anbeginn seiner Geschichte aktiv am Gestaltungs- und Kommunikationsprozess mitwirkt und die Kultur- und Menschheitsgeschichte prägt. ${ }^{6}$ Der Medienwissenschaftler Werner Faulstich (1998; id. 2002) grenzt dieses sogenannte Menschmedium (z. B. Erzähler*in, aber auch Feste, Tänze oder Hinrichtungsrituale) von Gestaltungs- (z. B. Statuen, Skulpturen, Denkmäler, Architektur) und Schreibmedien (z. B. Brief, Flugblatt, Zeitschrift) ab. Allerdings darf nicht vergessen werden, dass es auch der Mensch ist, der Gestaltungs- und Schreibmedien hervorbringt, und somit in der Rolle des Produzenten und Trägers nicht aufhört, Medium der Vermittlung von Erfahrungen ${ }^{7}$ und Ereignissen ${ }^{8}$ zu sein.

\subsection{NarRative Wissens- Und Welterzeugung ${ }^{9}$}

Insbesondere Mensch- und Schreibmedien verwenden die menschliche Sprache als arbiträres und konventionalisiertes Symbol- und Zeichensystem (Saussure), um zeitlich

6 Die grundlegende Bedeutung von zwischenmenschlicher Kommunikation für die Entstehung von individuellem wie kollektivem Bewusstsein und Gedächtnis - verstanden als Wissensbestände über sich selbst und die Gruppen, denen ein Mensch angehört - wurde in der Vergangenheit ausführlich hervorgehoben und in Konzepten wie denen der sozialen Rahmungen und des kollektiven Gedächtnisses (Halbwachs), des kulturellen und kommunikativen Gedächtnisses (Assmann, Welzer) oder der Erinnerungsorte (Nora) festgehalten.

7 Der Sozialwissenschaftler Alfred Schütz konzipiert den Unterschied zwischen ,erleben “ und ,erfahren' sowie die Transformation vom Erleben zum Erfahren folgendermaßen: Während des Vorgangs des Erlebens verarbeitet der Körper ständig sinnliche Eindrücke, die allerdings nicht bewusst wahrgenommen werden und nicht reflexiv zugänglich sind. Erleben ist somit ein vorreflexiver Vorgang, wie das Zuhören und Mitschreiben eines Vortrags. Wenn diese Erlebnisse zu einem Gedankeninhalt, also zu etwas bewusst Wahrgenommenem werden, über das nachgedacht und gesprochen werden kann, transformieren sie sich zu Erfahrungen (cf. Abels 2001, 62).

8 Aus narratologischer Sicht sind Ereignisse weder etwas Gegebenes noch etwas Natürliches, sondern vielmehr das Ergebnis bestimmter Arten der Welterzeugung, die mit Selektion, Löschung, Abstraktion und Priorisierung zu tun haben. Die Narratologie bietet eine Reihe nützlicher Kriterien, um den Begriff ,Ereignis‘ zu definieren, indem man ihn vom Begriff, Geschehen' abgrenzt und verschiedene Grade von ,Ereignishaftigkeit' unterscheidet. Anknüpfend an die alltägliche Bedeutung von Ereignis als ,signifikanter Vorfall` oder ,signifikantes Ereignis‘ unterscheidet die Erzähltheorie zunächst zwischen der Gesamtheit aller Ereignisse und dem Ereignis als besonders relevantem und wesentlichem Teil davon. Die Beschaffenheit eines Ereignisses rührt daher, dass es aus dem kontinuierlichen Fluss von Ereignissen herausgehoben wird und dadurch als etwas Besonderes oder Überraschendes qualifiziert wird: Es basiert also auf der Auswahl und Unterscheidung durch den/die Beobachter*in (cf. Hühn 2012; Nünning 2010, 197-199).

$9 \mathrm{Zu}$ den unterschiedlichen Positionen und Einschätzungen des Verhältnisses von Literatur, Kunst, Fotografie und Film zu Realität/Wirklichkeit vom 18. bis zum 21. Jahrhundert siehe 